MSC 35F20, 65D30

\title{
Estimates in the Taylor series method for polynomial total systems of PDEs
}

\author{
L. K. Babadzanjanz, I. Yu. Pototskaya, Yu. Yu. Pupysheva
}

St. Petersburg State University, 7-9, Universitetskaya nab., St. Petersburg, 199034, Russian Federation

For citation: Babadzanjanz L. K., Pototskaya I. Yu., Pupysheva Yu. Yu. Estimates in the Taylor series method for polynomial total systems of PDEs. Vestnik of Saint Petersburg University. Applied Mathematics. Computer Science. Control Processes, 2021, vol. 17, iss. 1, pp. 27-39. https://doi.org/10.21638/11701/spbu10.2021.103

Many of total systems of PDEs can be reduced to the polynomial form. As was shown by various authors, one of the best methods for the numerical solution of the initial value problem for ODE systems is the Taylor Series Method (TSM). In the article, the authors consider the Cauchy problem for the total polynomial PDE system, obtain the recurrence formulas for Taylor coefficients, and then formulate and prove a theorem on the accuracy of its solutions by TSM.

Keywords: Taylor Series Method, total polynomial PDE system, polynomial system, numerical PDE system integration.

1. Introduction. This work directly continues and generalizes what was proposed in the articles [1-6] (for ODE and PDE systems) and [7] (for total linear PDE systems) to the case of total polynomial systems of PDEs. First, we consider some preliminaries (largely from [1-7]): the Cauchy problem for total systems and polynomial total systems; additional variables method [2-4]; Taylor coefficients and estimates to total linear systems of PDEs; Cauchy formula for product of multivariate power series; the idea of schemes and the concept of the Taylor Series Method (TSM). In final section the examples of how one arrives at total polynomial systems of PDEs are discussed.

Remark 1. Note that using the additional variables method one reduces total systems of PDEs to polynomial form. Using the Cauchy formula of the product of multivariate power series and the method of undetermined coefficients we can derive simple recurrence formulas for Taylor coefficients. The derivation of these formulas is based on the concept of a scheme that was introduced in [2]: given the initial data and the scheme for right-hand sides of a polynomial total system, allows one to sequentially calculate all necessary Taylor coefficients of its solution.

As examples, the total polynomial ODE and PDE Cauchy problems one can consider in $[7,8]$.

2. Preliminaries to Cauchy problem for total PDEs. Consider the total system of partial differential equations with the initial conditions [9]:

$$
\frac{\partial x_{j}}{\partial t_{\nu}}=f_{\nu, j}\left(x_{1}, \ldots, x_{n}, t_{1}, \ldots, t_{s}\right), \quad x_{j}\left(t_{0}\right)=x_{0, j}, \quad j=1, \ldots, n, \quad \nu=1, \ldots, s .
$$

Methods for solving this problem are oriented to the general case when the right-hand sides $f_{\nu, j}$ belong to the class of smooth or piecewise smooth functions. At the same time, in many applied problems, for which methods are developed, it is quite possible to reduce this problem to the case when the functions $f_{\nu, j}$ are algebraic polynomials in $x_{1}, \ldots, x_{n}$

(C) St. Petersburg State University, 2021 
(by introducing the special additional variables [1-4]. In these cases, the obtained Cauchy problem is called polynomial, and it can be written as

$$
\frac{\partial x_{j}}{\partial t_{\nu}}=\sum_{m \in[0: L+1]} \sum_{i \in I(m)} a_{\nu, m, j}[i] x^{i}, \quad x_{j}\left(t_{0}\right)=x_{0, j}, \quad j=1, \ldots, n, \quad \nu=1, \ldots, s,
$$

or, in other form,

$$
\frac{\partial x_{j}}{\partial t_{\nu}}=\sum_{m=0}^{u} a_{\nu, m, j} x^{i(m)}, \quad x_{j}\left(t_{0}\right)=x_{0, j}, \quad j=1, \ldots, n, \quad \nu=1, \ldots, s,
$$

where

$$
\begin{gathered}
x=\left(x_{1}, \ldots, x_{n}\right) \in C^{n}, i=\left(i_{1}, \ldots, i_{n}\right) \Rightarrow x^{i}=x_{1}^{i_{1}} \cdot \ldots \cdot x_{n}^{i_{n}}, \\
x_{j}, x_{0, j}, t_{\nu}, t_{0, \nu}, a_{\nu, m, j} \in C, \quad x^{i(0)}=1, \quad|i|=i_{1}+\ldots+i_{n}, \\
I(m)=\left\{i \in Z^{n}\left|i_{1}, \ldots, i_{n} \geqslant 0,\right| i \mid=m\right\}, \quad L \in[0:+\infty), \quad u \in[1:+\infty),
\end{gathered}
$$

and $x^{i(0)}, x^{i(1)}, \ldots, x^{i(u)}$ are all different monomials from the right-hand sides of the equations (1) and (2).

Remark 2. The coefficients $a_{\nu, m, j}$ in (1) and (2), generally speaking, are different. Since the problems (1) and (2) are two forms of writing the same Cauchy problem, we use the one that seems more convenient, and each time we notice which form we are talking about, but this is also obvious in the context - by the formulas and from text.

We denote the solution of the problem (1) and (2) by $x\left(t, t_{0}, x_{0}\right)$ or, briefly, by $x(t)$. In addition, we will utilize the designations

$$
\begin{gathered}
x^{(k)}=\frac{\partial^{|k|} x}{\partial t^{k}}, \quad|k|=k_{1}+\ldots+k_{s}, \quad x_{0}^{(k)}=x^{(k)}\left(t_{0}\right), \\
x^{(0)}=x, \quad x_{0}^{(0)}=x_{0}, \quad|x|=\max _{i \in[1: n]}\left|x_{i}\right|, \quad O_{\rho}\left(t_{0}\right)=O_{\rho_{1}}\left(t_{0}\right) \times \ldots \times O_{\rho_{s}}\left(t_{0}\right), \\
O_{\rho_{\nu}}\left(t_{0}\right)=\left\{t \in C^{s}\left|(\forall j \in[1: s], j \neq \nu)\left(t_{j}=t_{0, j}\right),\right| t_{\nu}-t_{0, \nu} \mid<\rho_{\nu}\right\}, \\
T_{M} x\left(t, t_{0}, x_{0}\right)=\sum_{m=0}^{M} x_{0}^{(m)} \frac{\left(t-t_{0}\right)^{m}}{m !}, \quad \delta T_{M} x\left(t, t_{0}, x_{0}\right)=x\left(t, t_{0}, x_{0}\right)-T_{M} x\left(t, t_{0}, x_{0}\right), \\
m !=\prod_{\mu=1}^{s} m_{\mu} !, \quad 0 !=1, \quad k=\left(k_{1}, \ldots, k_{s}\right), \\
M=\left(M_{1}, \ldots, M_{s}\right) \in[0:+\infty)^{s}, \quad \rho=\left(\rho_{1}, \ldots, \rho_{s}\right) \in(0,+\infty)^{s} .
\end{gathered}
$$

Here $T_{M}$ and $\delta T_{M}$ are the operators that put in correspondence the Taylor polynomial $T_{M} x\left(t, t_{0}, x_{0}\right)$ and the remainder $\delta T_{M} x\left(t, t_{0}, x_{0}\right)$ to the solution of the problem (1) (or (2) which is the same). The vector radius of convergence of the Taylor series we denote as $R\left(t_{0}, x_{0}\right)=\left(R_{1}\left(t_{0}, x_{0}\right), \ldots, R_{s}\left(t_{0}, x_{0}\right)\right)$ and, instead, later in this paper as a domain, where Taylor series converge we will utilize $O_{\rho}\left(t_{0}\right)=O_{\rho_{1}}\left(t_{0}\right) \times \ldots \times O_{\rho_{s}}\left(t_{0}\right)$, see above in (3) and below - in Propositions 1 and 4.

2.1. Additional variables method and polynomial total systems of PDEs. In the case $s=1$, the idea of an above-mentioned reduction from the non-polynomial total Cauchy problem for PDEs (i. e. for ODEs) to the polynomial total problem (1) (or (2) which is the same) goes back to A. Poincare [10] and J. Steffensen [11, 12]. In 
the general case $s \geqslant 1$, in [1-4], sufficient and necessary and sufficient conditions for reducing equations to polynomial form were formulated and proved, numerous algorithms and examples from Dynamics and other fields of Mathematics and Mathematical Physics were also given there. We now give a couple of simple definitions, and then formulate the necessary and sufficient condition mentioned. A function $\varphi\left(x_{1}, \ldots, x_{\sigma}\right)$ is said to satisfy a total polynomial system, if it is a component of its solution. The class of all functions $\varphi\left(x_{1}, \ldots, x_{\sigma}\right)$ satisfying polynomial systems is denoted by $\Sigma_{\sigma}$. It is evident that the implications $\Sigma_{1} \subset \Sigma_{2} \subset \ldots$ hold true. All elementary functions and a wide variety of special functions of Mathematical Physics belong to $\Sigma_{1}$. By $F_{m}^{\sigma}, \sigma, m \in[1:+\infty)$, one denotes the class of all scalar functions of $x_{1}, \ldots, x_{m}$, which may be represented through finite compositions of basic operations,,$+- \times, /$ and functions belonging to $\Sigma_{\sigma}$. Of course, the basic operations can be considered as functions from $\Sigma_{\sigma}$, but then polynomials and rational functions would have to be denoted formally as a finite superpositions of functions from $\Sigma_{\sigma}$ which would be both funny and sad.

$\boldsymbol{N} \& \boldsymbol{S}$ Condition: The total system (0) one can reduce to polynomial form by introducing a number of additional variables if all $f_{\nu, j}$ belong to $F_{m}^{\sigma}$ for some $\sigma \in[1:+\infty)$.

2.2. Taylor coefficients and estimates to total linear systems of PDEs. Here we consider a special case of problem (2) (or (1)) - the linear Cauchy problem, which we write in the form:

$$
\begin{gathered}
\frac{\partial x}{\partial t_{\nu}}=a_{\nu}+A_{\nu} x, \quad x\left(t_{0}\right)=x_{0}, \quad \nu=1, \ldots, s, \\
x=\left(x_{1}, \ldots, x_{n}\right), \quad x_{0}=\left(x_{0,1}, \ldots, x_{0, n}\right) \in C^{n}, \\
a_{\nu}=\left(a_{\nu, 1}, \ldots, a_{\nu, n}\right) \in C^{n}, \quad\left|a_{\nu}\right|=\max _{i \in[1: n]}\left|a_{\nu, i}\right|, \\
t=\left(t_{1}, \ldots, t_{s}\right), \quad t_{0}=\left(t_{0,1}, \ldots, t_{0, s}\right) \in C^{s}, \quad A_{\nu}=\left(a_{\nu, i, j}\right), \quad a_{\nu, i, j} \in C .
\end{gathered}
$$

In addition to (3), we also need the given below notation here:

$$
\begin{gathered}
\left(A_{\nu}^{k_{\nu}} x\right)_{i}=\sum_{j=1}^{n} a_{\nu, i, j} x_{j}, \quad \rho_{\nu}=1 / s_{\nu}, \quad s_{\nu}=\left\|A_{\nu}\right\|_{\infty}=\max _{i \in[1: n]} s_{\nu, i}, \quad s_{\nu, i}=\sum_{j=1}^{n}\left|a_{\nu, i, j}\right|, \\
T_{\mu} e^{\tau}=\sum_{m=0}^{\mu} \frac{\tau^{m}}{m !}, \quad \delta T_{\mu} e^{\tau}=e^{\tau}-T_{\mu} e^{\tau}, \quad \mu=1,2, \ldots .
\end{gathered}
$$

Because of the equality

$$
\frac{\partial^{|k|} x}{\partial t^{k}}=\frac{\partial^{k_{\nu}} x}{\partial t^{k_{\nu}}}, \quad k=\left(k_{1}, \ldots, k_{s}\right), \quad|k|=k_{1}+\ldots+k_{s}, \quad \nu=1, \ldots, s,
$$

implies that the Taylor coefficients for solutions of the problem (4) satisfies the recurrence equalities

$$
\frac{x^{\left(k_{\nu}\right)}}{k_{\nu} !}=\frac{\left(A_{\nu}^{k_{\nu}} x+A_{\nu}^{k_{\nu}-1} a_{\nu}\right)}{k_{\nu} !} .
$$

Thus the result formulated in the following Proposition has been proved (see [7]).

Proposition 1. The solution $x\left(t, t_{0}, x_{0}\right)$ of the problem (4) is holomorphic on $O_{\rho_{\nu}}\left(t_{0}\right)$ (see $(3))$ separately in $t_{\nu}$ and satisfies there the inequality

$$
\left|\delta T_{M} x\left(t, t_{0}, x_{0}\right)\right| \leqslant\left(\left|x_{0}\right|+\left|a_{\nu}\right| \rho_{\nu}\right) \delta T_{M_{\nu}} e^{\left|t_{\nu}-t_{0, \nu}\right| / \rho_{\nu}} .
$$


The smaller $s_{\nu}=\rho_{\nu}^{-1}$ the better the estimates (6). To improve these estimates, it is natural to introduce a scaling transformation in the problem (4):

$$
x_{j}=\alpha_{j} y_{j}, \quad \alpha_{j}>0, \quad j \in[1: n] .
$$

Then instead of problem (4) we get the following:

$$
\begin{gathered}
\frac{\partial y}{\partial t_{\nu}}=b_{\nu}+B_{\nu} y, \quad y\left(t_{0}\right)=y_{0}, \quad \nu=1, \ldots, s, \\
y=\left(y_{1}, \ldots, y_{n}\right), \quad y_{0}=\left(y_{0,1}, \ldots, y_{0, n}\right), \quad b_{\nu}=\left(b_{\nu, 1}, \ldots, b_{\nu, n}\right), \quad B_{\nu}=\left(b_{\nu, i, j}\right), \\
y_{i}=\alpha_{i}^{-1} x_{i}, \quad b_{\nu, i}=\alpha_{i}^{-1} a_{\nu, i}, \quad b_{\nu, i, j}=\alpha_{i}^{-1} \alpha_{j} a_{\nu, i, j},
\end{gathered}
$$

and using the designations

$$
\rho_{\nu}(\alpha)=\frac{1}{s_{\nu}(\alpha)}, \quad s_{\nu}(\alpha)=\max _{i \in[1: n]} s_{\nu, i}(\alpha), \quad s_{\nu, i}(\alpha)=\alpha_{i}^{-1} \sum_{j=1}^{n} \alpha_{j}\left|a_{\nu, i, j}\right|, \quad \alpha=\left(\alpha_{1}, \ldots, \alpha_{n}\right)
$$

(compare (8) with (5)) have derived Corollary.

Corollary. The solution $x\left(t, t_{0}, x_{0}\right)$ of the problem (4) is holomorphic on $O_{\rho_{\nu}(\alpha)}\left(t_{0}\right)$ separately in $t_{\nu}$ and satisfies there the inequality

$$
\left|\delta T_{M} x_{i}\left(t, t_{0}, x_{0}\right)\right| \leqslant \alpha_{i}\left(\left|y_{0}\right|+\left|b_{\nu}\right| \rho_{\nu}(\alpha)\right) \delta T_{M_{\nu}} e^{\left|t_{\nu}-t_{0, \nu}\right| / \rho_{\nu}(\alpha)}
$$

(compare (9) with (6)).

As we noted above (see before (7)), to improve estimates (9), it is natural to reduce the value of $s_{\nu}(\alpha)$ by choosing $\alpha$. For the optimal choice of $\alpha$, it is necessary to solve a minimax problem. In [13], we gave examples of solving such problems for important real models described by ODE systems. Sometimes it's easier to select them as $\alpha$ for some other reasons. For example, you can use $\alpha$ obtained for the linear approximation of the original non-linear equations in a neighborhood of the initial data. In the paper [7], we explained how, in applications, the matrix $A_{\nu}$ of the mentioned approximation should be replaced with a square positive matrix $A_{\nu}^{+}$so that Perron's theorem [14] can be used to select scaling factors. Then as scaling factors $\alpha_{1}, \ldots, \alpha_{n}$ in Corollary it is natural to use the components of a positive eigenvector $\alpha^{*}=\left(\alpha_{1}^{*}, \ldots, \alpha_{n}^{*}\right)$ of the matrix $A_{\nu}^{+}$corresponding to its eigenvalue $\lambda\left(A_{\nu}^{+}\right)$, maximum in absolute value. For convenience, recall the Perron's theorem.

Theorem (Perron). Let the matrix $P=\left(p_{i, j}\right)$ be positive, $i$. e. $p_{i, j}>0$ for all $i, j \in[1: n]$. Then the following statements are true [10]:

a) there is a single eigenvalue $\lambda(P)$ of this matrix with the largest absolute value;

b) this eigenvalue is positive and simple, and the corresponding eigenvector can be chosen positive;

c) the following equality holds:

$$
\lambda(P)=\min _{x_{1}, \ldots, x_{n}>0} \max _{i \in[1: n]}\left(\sum_{j=1}^{n} p_{i, j} x_{j} / x_{i}\right) .
$$

2.3. Cauchy formula for product of multivariate power series. Consider the product of two series absolutely convergent in some complex domain:

$$
A=\sum_{\alpha_{1}, \ldots, \alpha_{s}=0}^{\infty} x_{\alpha}\left(t-t_{0}\right)^{\alpha}, \quad B=\sum_{\beta_{1}, \ldots, \beta_{s}=0}^{\infty} y_{\beta}\left(t-t_{0}\right)^{\beta},
$$




$$
\text { i. e. } A \cdot B=\sum_{\alpha_{1}, \ldots, \alpha_{s}=0}^{\infty} x_{\alpha}\left(t-t_{0}\right)^{\alpha} \sum_{\beta_{1}, \ldots, \beta_{s}=0}^{\infty} y_{\beta}\left(t-t_{0}\right)^{\beta} \text {. }
$$

This product is a series in powers of $\left(t-t_{0}\right)$ that converges absolutely in some domain $D \subset C^{s}$, and therefore, its sum does not depend on the order of the terms $x_{\alpha} x_{\beta}\left(t-t_{0}\right)^{\alpha+\beta}$. In particular, we can put

$$
\begin{gathered}
\sum_{\alpha=0}^{\infty} x_{\alpha}\left(t-t_{0}\right)^{\alpha} \cdot \sum_{\beta=0}^{\infty} y_{\beta}\left(t-t_{0}\right)^{\beta}=\sum_{\mu=0}^{\infty} \sum_{\alpha=0}^{\mu} x_{\alpha} y_{\mu-\alpha}\left(t-t_{0}\right)^{\mu} \\
\mu=\left(\mu_{1}, \ldots, \mu_{s}\right)=\alpha+\beta, \quad \alpha=\left(\alpha_{1}, \ldots, \alpha_{s}\right), \quad \beta=\left(\beta_{1}, \ldots, \beta_{s}\right) .
\end{gathered}
$$

2.4. Taylor series method to polynomial total systems of PDEs. The Taylor series method for solving the Cauchy problem (1) (or (2)) consists in constructing a table of approximate values $\tilde{x}_{t_{w}}=\tilde{x}\left(t_{w}\right)$ using the formula

$$
\tilde{x}_{\tau_{w}}=T_{N_{w}} x\left(\tau_{w}, \tau_{w-1}, \tilde{x}_{\tau_{w-1}}\right), \quad w=1,2, \ldots,
$$

where $N_{w}=\left(N_{w, 1}, \ldots, N_{w, s}\right) \in(0:+\infty)^{s}, \tau_{0}=t_{0}, \tau_{w}=\tau_{w-1}+h_{w}, \tau_{w}=\left(\tau_{w, 1}, \ldots, \tau_{w, s}\right)$, $h_{w}=\left(h_{w, 1}, \ldots, h_{w, s}\right) \in C^{s}$ and $h_{w}$ has to satisfy the inequalities

$$
\left|h_{w, \nu}\right|<R_{\nu}\left(\tau_{w-1}, \tilde{x}_{\tau_{w-1}}\right), \quad \nu=1, \ldots, s .
$$

The calculation of each value of $\tilde{x}_{\tau_{w}}$ is called the step of the method, and $h_{w}$ is called the size of this step (or, briefly, the step). In the general case of integration along a curve in $C^{s}$, all $h_{w, \nu}$ are complex numbers, and points $\tau_{w}$ lie on this curve. To calculate $\tilde{x}_{\tau_{w}}$ for some given $\tau_{w}$ with high accuracy even for $\tau_{w}$ from its domain of convergence (see (11)), the number of steps may turn out to be large, which can cause a fast accumulation of rounding errors and an increased processor time. That is why it is advisable to use the steps as large as possible (in actual fact, one has to find all $\rho_{\nu}$ as large as possible).

\section{Estimates in the case of polynomial total systems.}

3.1. Schemes and Taylor coefficients to polynomial systems of PDEs. Let the set $T=\left(x^{i(n+1)}, \ldots, x^{i(u)}\right)$ of all different nonlinear monomials in right-hand sides of system of equations (2) is arranged (as in equations (1)) in such a way that

$$
2 \leqslant|i(n+1)| \leqslant|i(n+2)| \leqslant \ldots \leqslant|i(u)| \leqslant L+1 .
$$

Here $|i|=i_{1}+\ldots+i_{n}$ is the power of the monomial $x^{i}$, and $L+1$ is the maximal power of monomials in $T$. Consider the condition

$$
(\forall r \in[(n+1): u))(\exists p, q \in[1: r))\left(x^{i(r)}=x^{i(p)} \cdot x^{i(q)}\right) .
$$

If this condition holds, then one can consider the scheme

$$
S=((p(n+1), q(n+1)), \ldots,(p(u), q(u)))
$$

consisting from $u-n$ pairs $(p(r), q(r))$ such that $r>p(r), q(r)$ for any $r \in[(n+1): u]$ (see $[2,6])$ : using the scheme one can propose algorithms which, given the monomials $x^{i(1)}=x_{1}, \ldots, x^{i(n)}=x_{n}$, implement successive evaluation of all other monomials in $T$.

It is obvious that any set of monomials can be supplemented by other monomials so that it has a scheme, and it can be assumed (and we do assume it in what follows) that 
the set $T$ of different non-linear monomials (supplemented, if necessary) has the scheme $S=((p(n+1), q(n+1)), \ldots,(p(u), q(u)))$.

In the case $s=1$, to calculate the Taylor coefficients $x_{k, p}$ of the solution

$$
x^{i(k)}=\sum_{p=0}^{\infty} x_{k, p}\left(t-t_{0}\right)^{p}, \quad k \in[0: u],
$$

to the problem (2) (or (1)) and all other monomials in $T$, we obtained recurrence formulas $[2]$

$$
\left.\begin{array}{c}
x_{k, 0}=x_{k}\left(t_{0}\right), \quad k \in[1: n], \\
x_{k, r}=\sum_{l=0}^{r} x_{p(k), l} x_{q(k),-l}, \quad k \in[n+1: u], \\
x_{k, r+1}=(r+1)^{-1} \sum_{l=0}^{u} a_{k, l} x_{l, r}, \quad k \in[1: n]
\end{array}\right\}, r=0,1, \ldots .
$$

Now, acting in a similar (but slightly more complicated) way, we generalize these formulas to the case $s \geqslant 1$ (see below - (18), (19)). If we assume that (see (2))

$$
x^{i(m)}=\sum_{l_{1}, \ldots, l_{s}=0}^{\infty} x_{m, l}\left(t-t_{0}\right)^{l}, \quad m=0, \ldots, u, \quad l=\left(l_{1}, \ldots, l_{s}\right),
$$

then using the method of undetermined coefficients, one can derive recurrent formulas to the Taylor coefficients $x_{m, l}=x_{m, l_{1}, \ldots, l_{s}}$. Indeed, substituting (15) into the equation contained within the condition (12) and using the Cauchy formula (10) we obtain:

$$
\begin{gathered}
x^{i(r)}=x^{i(p)} \cdot x^{i(q)}, \quad r \in[(n+1): u), \quad p(r), \quad q(r) \in[1: r) \Rightarrow \\
\Rightarrow \sum_{l=0}^{\infty} x_{r, l}\left(t-t_{0}\right)^{l}=\sum_{l=0}^{\infty} x_{p(r), l}\left(t-t_{0}\right)^{l} \cdot \sum_{l=0}^{\infty} x_{q(r), l}\left(t-t_{0}\right)^{l}= \\
=\sum_{l=0}^{\infty} \sum_{\mu=0}^{l} x_{p(r), l} x_{q(r), l-\mu}\left(t-t_{0}\right)^{l}, \quad \mu=\left(\mu_{1}, \ldots, \mu_{s}\right) \Rightarrow \\
\Rightarrow \sum_{l=0}^{\infty}\left(x_{r, l}-\sum_{\mu=0}^{l} x_{p(r), l} x_{q(r), l-\mu}\right)\left(t-t_{0}\right)^{l}=0 \Rightarrow x_{r, l}-\sum_{\mu=0}^{l} x_{p(r), l} x_{q(r), l-\mu}=0 .
\end{gathered}
$$

Then substituting (15) into (2) and using the designations $e_{1}=(1,0, \ldots, 0), \ldots, e_{s}=$ $(0, \ldots, 0,1)$ we derive:

$$
\begin{gathered}
\partial \sum_{\lambda_{1}, \ldots, \lambda_{s}=0}^{\infty} x_{j, \lambda}\left(t-t_{0}\right)^{\lambda} / \partial t_{\nu}=\sum_{k=0}^{u} a_{\nu, j, k} x^{i(k)}, \quad j=1, \ldots, n, \Rightarrow \\
\Rightarrow \quad \sum_{\lambda=\left(\lambda_{1}, \ldots, \lambda_{s}\right)=0}^{\infty} \lambda_{\nu} x_{j, \lambda}\left(t-t_{0}\right)^{\lambda-e_{\nu}}=\sum_{k=0}^{u} a_{\nu, j, k} \sum_{l_{1}, \ldots, l_{s}=0}^{\infty} x_{k, l}\left(t-t_{0}\right)^{l} .
\end{gathered}
$$

Making the substitution $\lambda \mapsto\left(l+e_{\nu}\right)$ in the left-hand side of this formula, we obtain:

$$
\sum_{l_{1}, \ldots, l_{s}=0}^{\infty}\left(l_{\nu}+1\right) x_{j, l+e_{\nu}}\left(t-t_{0}\right)^{l}=\sum_{k=0}^{u} a_{\nu, j, k} \sum_{l_{1}, \ldots, l_{s}=0}^{\infty} x_{k, l}\left(t-t_{0}\right)^{l} \Rightarrow
$$




$$
\begin{gathered}
\Rightarrow \sum_{l_{1}, \ldots, l_{s}=0}^{\infty}\left[\left(l_{\nu}+1\right) x_{j, l+e_{\nu}}-\sum_{k=0}^{u} a_{\nu, j, k} x_{k, l}\right]\left(t-t_{0}\right)^{l}=0 \Rightarrow \\
\Rightarrow x_{j, l+e_{\nu}}=\frac{1}{l_{\nu}+1} \sum_{k=0}^{u} a_{\nu, j, k} x_{k, l} .
\end{gathered}
$$

Formulas (2), (16), (17) and designations

$$
l^{r}=\left(l_{1}^{r}, \ldots, l_{s}^{r}\right), \quad l^{r+1}=\left(l_{1}^{r}+1, \ldots, l_{s}^{r}+1\right), \quad l^{0}=(0, \ldots, 0), \quad r=0,1,2, \ldots,
$$

lead to the following algorithm for sequentially calculating the Taylor coefficients to the solution of the Cauchy problem to the total polynomial system of partial differential equations (2) (and (1)):

$$
\left.\begin{array}{c}
x_{j, 0}=x_{j}\left(t_{0}\right), \quad j \in[1: n], \\
x_{j, l^{r}}=\sum_{\mu=0}^{l^{r}} x_{p(j), l^{r}} x_{q(j), l^{r}-\mu}, \quad j \in[(n+1): u], \\
x_{j, l^{r}+e_{\nu}}=\frac{1}{l_{\nu}+1} \sum_{k=0}^{u} a_{\nu, j, k} x_{k, l}, \quad j \in[1: n], \quad \nu \in[1: s]
\end{array}\right\}, \quad r=0,1, \ldots .
$$

Note that in the case $\mathrm{s}=1$, these formulas reduce to (13), (14).

The estimates for linear equations one can find in our paper [7].

\subsection{Introduction to the infinite systems method to polynomial systems of} $\boldsymbol{P D E s}$. Omitting details, the idea of the method of infinite systems can be outlined as follows $[1,2]$.

$S$ t e $p$ 1. Introducing an infinite set of new variables, one reduces the original problem (1) or (2) to the Cauchy problem for an infinite linear system of differential equations.

$S$ t e $p$ 2. Using linearity, some results are obtained for the last problem.

$S$ t e $p$ 3. These results are interpreted in terms of the original Cauchy problem.

In accordance with this idea, let us, for any $i \in \bigcup_{m=1}^{+\infty} I(m)$, introduce the variables in the problems (1), (2)

$$
x[i]=x^{i},
$$

and suppose that the sets $\chi(m)=\{x[i] \mid i \in I(m)\}$ are ordered in increasing $m$, and the elements in each of them are ordered so that $i_{1}=k_{1}, \ldots, i_{j}=k_{j}, i_{j+1}>k_{j+1}$ implies that $x[i]$ precedes $x[k]$. The ordered variables $x[i]$ are denoted by $z_{1}, z_{2}, \ldots$ and the vector of these is $z=\left(z_{1}, z_{2}, \ldots\right)$. In addition, we introduce the initial value

$$
z_{0}=\left(z_{1,0}, z_{2,0}, \ldots, z_{n, 0}, z_{n+1,0}, \ldots\right)=\left(x_{1,0}, x_{2,0}, \ldots, x_{n, 0}, x_{1,0}^{2}, x_{1,0} x_{2,0}, \ldots, x_{n, 0}^{2} \ldots\right)
$$

respectively corresponding to the initial vector $x_{0}=\left(x_{1,0}, x_{2,0}, \ldots, x_{n, 0}\right)$ of the original Cauchy problem. The numbers $p$ of the components $z_{p}$ of $z$ are associated with multiindices $i \in \bigcup_{m=1}^{+\infty} I(m)$ of variables $x[i]$ by some one-to-one correspondence $p=\omega(i)$, $i=\Omega(p)\left(\Omega=\omega^{-1}\right)$, which has the obvious property

$$
\omega\left(\chi_{q}\right)=\left(\sigma_{q-1}(n): \sigma_{q}(n)\right],
$$

where $\sigma_{q}(n)=(q+n) ! /(q ! n !)-1$ is the number of elements in the set $\bigcup_{m=1}^{q} \chi_{m}$.

Proposition 2. If we assume that $a_{\nu, j, m}[i]$ in (1) are defined for all multi-indices $i$ with integer components, moreover, they are equal to zero, if $|i|>L+1$ or at least one of the components of $i$ is negative, then the following formulas hold: 


$$
\begin{gathered}
\frac{\partial x[k]}{\partial t_{\nu}}=\sum_{m=0}^{L} \sum_{i \in J(m)} \alpha_{\nu, m}[k, i] x[k+i], \quad \nu=1, \ldots, s, \\
k=\left(k_{1}, \ldots, k_{n}\right) \in Z^{n}, \quad z\left(t_{0}\right)=z_{0} .
\end{gathered}
$$

Here

$$
J(m)=\left\{i \in Z^{n}\left|i_{1} \geqslant-1, \ldots, i_{n} \geqslant-1,\right| i \mid=m\right\}, \quad \alpha_{\nu, m}[k, i]=\sum_{j=1}^{n} k_{j} a_{\nu, j, m}\left[i+e_{j}\right] .
$$

P r o o f. Indeed,

$$
\begin{gathered}
\frac{\partial x[k]}{\partial t_{\nu}}=\frac{\partial\left(x_{1}^{k_{1}} \cdot \ldots \cdot x_{n}^{k_{n}}\right)}{\partial t_{\nu}}=\sum_{j=1}^{n} \frac{k_{j}\left(x^{k} / x_{j}\right) \partial x_{j}}{\partial t_{\nu}}= \\
=\sum_{j=1}^{n} k_{j}\left(x^{k} / x_{j}\right) \sum_{m=1}^{L+1} \sum_{i \in I(m)} a_{\nu, j, m}[i] x^{i}=\sum_{j=1}^{n} \sum_{m=0}^{L+1} \sum_{i \in I(m)} k_{j} a_{\nu, j, m}[i] x^{k+i} / x_{j}= \\
=\sum_{m=0}^{L} \sum_{i \in J(m)}\left(\sum_{j=1}^{n} k_{j} a_{\nu, j, m}\left[i+e_{j}\right]\right) x[k+i]=\sum_{m=0}^{L} \sum_{i \in J(m)} \alpha_{\nu, m}[k, i] x[k+i],
\end{gathered}
$$

which is the required result.

3.3. Estimates to polynomial systems of PDEs. Let us write $(22)$ as the $s$ Cauchy problems for the function $z$ :

$$
\frac{\partial z}{\partial t_{\nu}}=G_{\nu} z, \quad \nu=1, \ldots, s, \quad z\left(t_{0}\right)=z_{0},
$$

and note that in fact these (like (22)) are $s$ separate countable Cauchy problems of the same structure, differing in matrices $G_{\nu}$ and arguments $t_{\nu}$. Each row of the matrix $G_{\nu}$ and each of its columns contain only a finite number of nonzero elements. Therefore, in particular, all its non-negative degrees are defined. Differentiating successively equation (23) we obtain

$$
\frac{\partial z}{\partial t_{\nu}}=G_{\nu} z, \quad \frac{\partial^{2} z}{\partial t_{\nu}^{2}}=G_{\nu} G_{\nu} z=G_{\nu}^{2} z, \ldots, \frac{\partial^{r} z}{\partial t_{\nu}^{r}}=G_{\nu}^{r} z, \ldots
$$

Now we use formulas (22) to obtain estimates of the quantities (24).

Proposition 3. Let $a_{\nu, j, m}[i], x_{0,1}, \ldots, x_{0, n}$ be the coefficients and initial data of problem (1), $G_{\nu}$ be the matrix of equation $(23),\left(G_{\nu} z\right)_{p}$ be the $p$-th component of the vector $G_{\nu} z$ and the following designations are used:

$$
s_{\nu}=\max _{j \in[1: n]} s_{\nu, j}, \quad s_{\nu, j}=\sum_{m=0}^{L} \gamma^{m} \sum_{i \in J(m)}\left|a_{\nu, j, m}\left[i+e_{j}\right]\right|, \quad \gamma=\left|x_{0}\right|=\max _{j \in[1: n]}\left|x_{0, j}\right| .
$$

Then for any natural numbers $p, q$ connected by the relation (see (21))

$$
p \in\left(\sigma_{q-1}(n): \sigma_{q}(n)\right],
$$


and for any natural number $j$, the inequality

$$
\left|\left(G_{\nu}^{j} z\right)_{p}\right| \leqslant s^{j} \gamma^{q} \prod_{m \in[0: j-1]}(q+m L), \quad \nu=1, \ldots, s, \quad \gamma=\left|x_{0}\right|=\max _{l \in[1: n]}\left|x_{0, l}\right|,
$$

holds.

$\mathrm{P}$ r o o f. We will use mathematical induction.

1. From definition (20) and $\gamma($ see $(25))$ we deduce the inequality

$$
|x[k+i]|=|x[k]||x[i]| \leqslant \gamma^{k} \gamma^{i} .
$$

If $p, q$ are connected by relation (26), then this means that $z_{p}=x[k]$ for $|k|=q$, and, using (22), (27), (28), we have the following inequalities:

$$
\left|\left(G_{\nu} z\right)_{p}\right|=\left|\frac{\partial z_{p}}{\partial t_{\nu}}\right|=\left|\frac{\partial x[k]}{\partial t_{\nu}}\right| \leqslant \sum_{m=0}^{L} \sum_{i \in J(m)} \sum_{j=1}^{n} k_{j}\left|a_{\nu, j, m}\left[i+e_{j}\right] \| x[k+i]\right| \leqslant s \gamma^{q} q,
$$

this means that inequality (27) is proved for $j=1$.

2 . Suppose that $p, q$, as before, are connected with the relation (26) and the inequality (27) holds for $j=r$. Let the designations $y_{p}=d^{r} z_{p} / d t_{\nu}^{r}, y[k]=d^{r} x[k] / d t_{\nu}^{r}, y=\left(y_{1}, y_{2}, \ldots\right)$ are introduced. As one can see, this means that $y_{p}=y[k], y=d^{r} z / d t_{\nu}^{r}$ and $y=G_{\nu}^{r} z$ (see $(27)$ ). Recalling the definition of the function $\omega$ (see (21)) one derives the inequality

$$
|y[k+i]|=\left|y_{\omega(k+i)}\right|=\left|\left(G_{\nu}^{r} z\right)_{\omega(k+i)}\right| \leqslant s^{r} \gamma^{|k+i|} \prod_{m \in[0: r-1]}(|k+i|+m L) .
$$

Differentiating (22) and (23) $r$ times, one obtains similar equations

$$
\begin{gathered}
\frac{\partial y[k]}{\partial t_{\nu}}=\sum_{m=0}^{L} \sum_{i \in J(m)} \alpha_{\nu, m}[k, i] y[k+i], \\
\frac{\partial y}{\partial t_{\nu}}=G_{\nu} y .
\end{gathered}
$$

Using (29)-(31) one obtains:

$$
\begin{gathered}
\left|\left(G_{\nu}^{r+1} z\right)_{p}\right|=\left|\left(G_{\nu} y\right)_{p}\right|=\left|\frac{\partial y_{p}}{\partial t_{\nu}}\right|=\left|\frac{\partial y[k]}{\partial t_{\nu}}\right| \leqslant \sum_{m=0}^{L} \sum_{i \in J(m)} \sum_{j=1}^{n} k_{j}\left|a_{\nu, j, m}\left[i+e_{j}\right]\right||y[k+i]| \leqslant \\
\leqslant \sum_{m=0}^{L} \sum_{i \in J(m)} \sum_{j=1}^{n} k_{j}\left|a_{\nu, j, m}\left[i+e_{j}\right]\right| s^{r} \gamma^{|k+i|} \prod_{\mu \in[0: r-1]}(|k+i|+\mu L) \leqslant \\
\leqslant s^{r+1} \gamma^{q} q \prod_{\mu \in[0: r-1]}(q+L+\mu L)=s^{r+1} \gamma^{q} \prod_{\mu \in[0: r]}(q+\mu L),
\end{gathered}
$$

i. e., inequality (27) holds for $j=r+1$. Thus, the inequality (27) is proved by induction.

Proposition 4. Let $a_{\nu, m, j}, x_{0,1}, \ldots, x_{0, n}$ be the coefficients and initial data of the problem (1) and, in addition to (3), the following designations are used:

$$
\rho_{\nu}=1 /\left(L s_{\nu}\right), \quad s_{\nu}=\max _{j \in[1: n]} s_{\nu, j}
$$




$$
\begin{gathered}
s_{\nu, j}=\sum_{m=0}^{L} \gamma^{m} \sum_{i \in J(m)}\left|a_{\nu, m, j}\left[i+e_{j}\right]\right|, \quad \gamma=\left|x_{0}\right|=\max _{j \in[1: n]}\left|x_{0, j}\right|, \\
b(\tau)=(1-\tau)^{-1 / L}, \quad b_{0}^{(m)}=\left(\frac{d^{m} b(\tau)}{d \tau^{m}}\right)_{\tau=0}=\prod_{l=0}^{m-1}(1 / L+l) .
\end{gathered}
$$

Then, for every $\nu \in[1: s], j \in[1:+\infty), M=\left(M_{1}, \ldots, M_{s}\right) \in[1:+\infty)^{s}$ the solution $x\left(t, t_{0}, x_{0}\right)$ of the problem (1) is holomorphic on $O_{\rho_{\nu}}\left(t_{0}\right)$ (see $\left.(3)\right)$ separately in $t_{\nu}$ and satisfies there the inequality

$$
\left|\delta T_{M} x\left(t, t_{0}, x_{0}\right)\right| \leqslant\left|x_{0}\right| \delta T_{M} b\left(\left|t-t_{0}\right| / \rho_{\nu}\right) .
$$

$\mathrm{P}$ r o o f. The set of the first $n$ components of the solution to the problem (23) coincides with the solution to the problem (1); therefore, from (24) and (27) (for $q=1$, $p \in[1: n])$ we obtain

$$
\left|x_{0}^{(l)}\right| \leqslant s^{l} \gamma \prod_{m \in[0: l-1]}(l+m L) .
$$

Using this inequality, we find

$$
\begin{gathered}
\left|\delta T_{M} x\left(t, t_{0}, x_{0}\right)\right|=\left|\sum_{l=M+1}^{\infty} x_{0}^{(l)} \frac{\left(t-t_{0}\right)^{l}}{l !}\right| \leqslant \\
\leqslant\left|x_{0}\right| \sum_{l=M+1}^{\infty} x_{0}^{(l)}\left(\frac{t-t_{0}}{\rho}\right)^{l} \prod_{m \in[0: l-1]} \frac{1 / L+m}{l !}=\left|x_{0}\right| \delta T_{M} b\left(\frac{t-t_{0}}{\rho}\right) .
\end{gathered}
$$

Q.E.D.

Now, introducing the additional variable $x_{n+1}=1$, replacing the free terms $a_{\nu, 0, j}$ in (1) with $a_{\nu, 0, j} x_{n+1}$, and using there the scaling transformation $x_{j}=\alpha_{j} y_{j}, j \in[1: n]$, where $\alpha_{j}$ are arbitrary positive parameters we can see that Proposition 4 implies the following result (compare with Corollary).

Proposition 5. Let $a_{\nu, m, j}, x_{0}=\left(x_{0,1}, \ldots, x_{0, n}\right)$, be the coefficients and initial data of the problem (1) and, in addition to (3), the following designations are used:

$$
\begin{gathered}
\gamma=\left|x_{0}\right|=\max _{j \in[1: n]} s\left|x_{0, j}\right|, \quad \rho_{\nu}(\alpha)=\frac{1}{L s_{\nu}(\alpha)}, \\
s_{\nu}(\alpha)=\max _{j \in[1: n]} s_{\nu, j}(\alpha), \quad s_{\nu, j}(\alpha)=\alpha_{j}^{-1}\left(\left|a_{\nu, 0, j}\right|+\sum_{m=0}^{L} \gamma^{m} \sum_{i \in J(m)}\left|a_{\nu, m, j}\left[i+e_{j}\right]\right|\right), \\
b(\tau)=(1-\tau)^{-1 / L}, \quad b_{0}^{(m)}=\left(\frac{d^{m} b(\tau)}{d \tau^{m}}\right)_{\tau=0}=\prod_{l=0}^{m-1}(1 / L+l) .
\end{gathered}
$$

Then, for every $\nu \in[1: s], j \in[1:+\infty), M=\left(M_{1}, \ldots, M_{s}\right) \in[1:+\infty)^{s}$, the solution $x\left(t, t_{0}, x_{0}\right)$ of the problem (1) is holomorphic on $O_{\rho_{\nu}}\left(t_{0}\right)$ (see $\left.(3)\right)$ separately in $t_{\nu}$ and satisfies there the inequality

$$
\left|\delta T_{M} x\left(t, t_{0}, x_{0}\right)\right| \leqslant\left|x_{0}\right| \delta T_{M} b\left(\left|t-t_{0}\right| / \rho\right) .
$$


4. Conclusion. In Section 2 of this paper the systems of PDEs in general and two different polynomial forms were considered. Besides, some necessary questions such as reduction of DEs in Dynamics to polynomial form by introducing a number of additional variables (the necessary and sufficient conditions), the idea of schemes and the concept of the TSM, obtaining Taylor coefficients and estimates to total linear and polynomial systems of PDEs, Cauchy formula for product of multivariate power series, necessary notation etc. The main results we considered in the Section 3. There using the infinite systems method we formulated and proved the Propositions 2-5. The last proposition one can consider as a real means of implementing the Taylor series method of solving polynomial systems of total partial differential equations. It is worth noting that the beginning of the research presented in the article were the papers on which the dissertation $[15]$ is based.

The prospects. As a short-term perspective, we suggest the proposed method applying to important problems in Mathematical Physics, Astronomy, Dynamics, Chemistry, and Applied Mathematics in general. In particular, it worth to be considered the following tasks:

- four examples of total polynomial systems of partial differential equations for the two-body problem proposed by us in [7];

- a series of systems of total Dynamics equations, the right-hand sides of which depend on different force potentials (see, for example, [16-20]).

\section{References}

1. Babadzanjanz L. K. Suschestvovaniye prodolgeniya i predstavleniye resheniya v nebesnoy mechanike [Existence of the continuations and representation of the solutions in celestial mechanics]. Trudy ITA AN SSSR [Proceedings of the ITA AS USSR], 1978, vol. XVII, pp. 3-45. (In Russian)

2. Babadzanjanz L. K. Metod ryadov Taylora [The Taylor series method]. Vestnik of Saint Petersburg University. Series 10. Applied Mathematics. Computer Science. Control Processes, 2010, iss. 3, pp. 13-29. (In Russian)

3. Babadzanjanz L. K. Metod dopolnitelnih peremennih [The additional variables method]. Vestnik of Saint Petersburg University. Series 10. Applied Mathematics. Computer Science. Control Processes, 2010, iss. 4, pp. 3-11. (In Russian)

4. Babadzanjanz L. K., Bregman K. M. Algorithm metoda dopolnitelnih peremennih [Algorithm of the additional variables method]. Vestnik of Saint Petersburg University. Series 10. Applied Mathematics. Computer Science. Control Processes, 2012, iss. 2, pp. 3-12. (In Russian)

5. Babadzanjanz L. K., Bol'shakov A. I. Primenenie metoda ryadov Taylora dlya resheniya obiknovennih differentsialnih uravneniy [Implementation of the Taylor series method for solving ordinary differential equations]. Vychislitelnye Metody i Programmirovanie [Numerical Methods and Programming], 2012, vol. 13, iss. 4, pp. 497-510. (In Russian)

6. Alesova I. M., Babadzanjanz L. K., Pototskaya I. Yu., Pupysheva Yu. Yu., Saakyan A. T. Highprecision numerical integration of equations in dynamics. International scientific conference on mechanics "The Eighth Polyakhov's Reading", Saint Petersburg, 2018, AIP Conference Proceedings, 2018, vol. 1959, no. 080005. https://doi.org/10.1063/1.5034722

7. Babadzanjanz L. K., Pototskaya I. Yu, Pupysheva Yu. Yu. Estimates for Taylor series method to linear total systems of PDEs. Vestnik of Saint Petersburg University. Series 10. Applied Mathematics. Computer Science. Control Processes, 2020, vol. 16, iss. 1, pp. 3-11.

8. Babadzanjanz L. K., Bregman A. M., Bregman K. M., Kasikova P. V., Petrosyan L. A. Polniye systemy uravneniy $\mathrm{v}$ zadache dvuh tel [Total systems of equations to the two-body problem]. Engineering sciences - from theory to applications. Coll. papers on LXI Internanional Conference. Novosibirsk, SIBAK Publ., 2016, vol. 8(56), pp. 13-21. (In Russian)

9. Gaishun I. V. Vpolne razreshimiye mnogomerniye differentsialniye uravneniya [Completely solvable multidimensional differential equations]. Minsk, Belarus, Nauka i technika Publ., 1983, 272 p. (In Russian)

10. Poincare H. Memoire sur les courbes definies par une equation differentielle. Journal de mathematiques pures et appliquees, 1886, series 4, vol. 2, pp. 151-218. 
11. Steffensen J. F. On the restricted problem of three bodies. Mat.-Fys. Medd. Danske, Viden-skab., Selskab, 1956, vol. 30, no. 18, pp. 75-83.

12. Steffensen J. F. On the problem of three bodies in the plane. Mat.-Fys. Medd. Danske, Videnskab., Selskab, 1957, vol. 31. no. 3. pp. 98-123.

13. Babadzanjanz L. K., Pototskaya I. Yu., Pupysheva Yu. Yu. Error estimates for numerical integration of ODEs in the minimax formulation. 2017 Constructive Nonsmooth Analysis and Related Topics (Dedicated to the memory of V. F. Demyanov), CNSA 2017, Proceedings. Saint Petersburg, Russia, 2017. https://doi.org/10.1109/CNSA.2017.7973932

14. Bellman R. Introduction to matrix analysis. 2nd ed. Philadelphia, SIAM Publ., 1997, 403 p.

15. Babadzanjanz L. K. Method beskonechnih system v zadachah nebesnoy mechaniki [Infinite system method in celestial mechanics problems]. Dis. of Dr. Sci. in Physics and Mathematics. Saint Petersburg, Saint Petersburg State University Publ., 1985, 236 p. Available at:

http://www.apmath.spbu.ru/ru/staff/babadzhanyants/publ/publ27.pdf (accessed: November 01, 2020). (In Russian)

16. Antonov V. A. Elementy teorii gravitatsionnogo potentsiala $i$ necotoriye sluchayi ego yavnogo vyrageniya [Elements of the theory of gravitational potential and some cases of its explicit expression]. Saint Petersburg, Saint Petersburg State University Press, 2008, 208 p. (In Russian)

17. Antonov V. A. Vvedeniye $v$ teoriyu nyutonovskogo potentsiala [Introduction to the theory of Newtonian potential]. Moscow, Nauka Publ., 1988, 270 p. (In Russian)

18. Kondratiev B. P. Teoriya potentsiala (noviye metodi $i$ zadachi s recheniyami) [Potential theory (New methods and problems with solutions)]. Moscow, Mir Publ., 2007, 512 p. (In Russian)

19. Sretensky L. N. Teoriya nyutonovskogo potentsiala [Theory of Newtonian potential]. Moscow, Leningrad, Gostechizdat Publ., 1946, 322 p. (In Russian)

20. Uchaykin V. V. Mechanika. Osnovy mekhaniki sploshnyh sred. Zadachi i upragnenia [Mechanics. Fundamentals of continuum mechanics. Tasks and exercises]. Moscow, Ijevsk, Institute of computer science Publ., 2002, 179 p. (In Russian)

Received: November 03, 2020.

Accepted: January 15, 2021.

Authors' information:

Levon K. Babadzanjanz - Dr. Sci. in Physics and Mathematics, Professor; levon-lkb@yandex.ru

Irina Yu. Pototskaya - PhD in Physics and Mathematics, Associate Professor; irinapototskaya@yandex.ru

Yulia Yu. Pupysheva - PhD in Physics and Mathematics, Associate Professor; j_poupycheva@mail.ru

\title{
Оценки в методе рядов Тейлора для полиномиальных полных систем УрЧП
}

\author{
Л. К. Бабаджаняни, И. Ю. Поточкая, Ю. Ю. Пупьшева
}

Санкт-Петербургский государственный университет, Российская Федерация, 199034, Санкт-Петербург, Университетская наб., 7-9

Для цитирования: Babadzanjanz L. K., Pototskaya I. Yu., Pupysheva Yu. Yu. Estimates for Taylor series method to polynomial total systems of PDEs // Вестник Санкт-Петербургского университета. Прикладная математика. Информатика. Процессы управления. 2021. Т. 17. Вып. 1. C. 27-39. https://doi.org/10.21638/11701/spbu10.2021.103

Многие из полных систем уравнений в частных производных сводятся к полиномиальной форме. Как было показано разными авторами, одним из лучших методов численного решения задачи начального приближения для систем обыкновенных дифференциальных уравнений является метод рядов Тейлора (TSM). В предыдущих статьях авторов была рассмотрена задача Коши для линейных полных систем УрЧП, получены коэффициенты Тейлора и оценки для методов рядов Тейлора для линейного случая. На основе этих результатов в настоящей работе рассматривается задача Коши для полной 
полиномиальной системы УрЧП. Для этого случая получены рекуррентные формулы для коэффициентов Тейлора. С их помощью сформулирована и доказана теорема о точности решения полной полиномиальной системы УрЧП методом TSM с использованием метода бесконечных систем.

Ключевые слова: метод рядов Тейлора, полные полиномиальные системы УрЧП, полиномиальные системы, численное интегрирование систем УрЧП.

Контактная информация:

Бабаджсаняни, Левон Константинович - д-р физ.-мат. наук, проф.; levon-lkb@yandex.ru

Поточкая Ирина Юръевна - канд. физ.-мат. наук, доц.; irinapototskaya@yandex.ru

Пупышева Юлия Юръевна - канд. физ.-мат. наук, доц.; j_poupycheva@mail.ru 